\title{
SU ALCUNI PRINCIPI DELLA GRAMMATICOGRAFIA LATINO-VOLGARE: I FRAMMENTI GRAMMATICALI LATINO-FRIULANI
}

Questo saggio è offerto al nostro amico Pavao Tekavčić come omaggio all'opera sua, d'importanza fondamentale nei campi della grammatica italiana e della linguistica reto-romanza e balcano-romanza. Come argomento ho scelto i frammenti grammaticali della seconda metà del secolo XIV provenienti da Friuli. Quei frammenti ${ }^{1}$, che lo Schiaffini aveva pubblicati nel $1921-1922^{2}$, si inseriscono in un quadro d'istruzione grammaticale elementare per il quale abbiamo testimonianze non solo nel nord d'Italia ${ }^{3}$, ma anche nel dominio d'oïl' .

Il tipo di grammatiche latino-volgari dei secoli XIII-XIV si presenta, in genere, come una sinopsi della morfologia latina secondo il modello dell'Ars minor di Donato e come una presentazione sommaria di costruzioni verbali in latino, secondo il modello del Doctrinale puerorum d'Alessandro di Villadei. L'esposizione si fa con intento didattico specifico: l'allievo deve assimilare le costruzioni di verbi latini non in astratto, $\mathrm{ma}$ in connessione con i verbi nel volgare (dei quali non si presenta la co-

1 I frammenti provengono da Cividale, un importante centro di studi grammaticali nel Medio Evo; cf. G. Tiraboschi, Storia della letteratura italiana V, I, Roma, 1807, p. 91; P.S. Leicht, "Il primo tentativo di costituire un' Università nella Venezia orientale“, Memorie storiche forogiuliesi 6, 1910, 1-14; R. Salaman, "Eine vergessene Universitätsgründung", Neues Archiv der Gesellschaft für ältere deutsche Geschichtskunde 36, 1911, 810-817, 879-880.

2 A. Schiaffini, "Frammenti grammaticali latino-friulani del secolo XIV", Rivista della Società filologica friulana 2, 1921, 3-16,93-105; ID. "Esercizi di versione dal volgare friulano in latino nel sec. XIV in una scuola notarile cividalese“, Rivista della Società filologica friulana 3, 1922, 87-117.

3 Si veda, ad esempio, le edizioni dei frammenti per: R. Sabbadini, "Frammenti di grammatica latinobergamasca", Studi medievali 1, 1904-1905, 281-292; A. De Stefano, "Una nuova grammatica latino-italiana del secolo XIII", Revue des langues romanes 48, 1905, 495-529; C. Marchesi, "Due grammatici del Medio Evo“, Bulletino della Società filologica romana 12, 1910, 19-56; G. Manacorda, "Un testo di grammatica latino-veneta del sec. XIII", Atti della R. Accademia di Scienze di Torino 49, 1913-1914, 689-698.

4 Si veda S. Heinimann, "L'Ars minor de Donat traduit en ancien français", Cahiers Ferdinand de Saussure 23, 1966, 49-59; M. Colombo Timelli, Un rifacimento antico-francese dell'Ars minor $d i$ Donato: il manoscritto Parigi B.N. lat. 14095, Milano, 1988; Th. Städtler, Zu den Anfängen der französischen Grammatiksprache, Tübingen, 1988; B. Merrilees, "Teaching Latin in French: Adaptations of Donatus' Ars minor", Fifteenth-Century Studies 12, 1987, 87-98; ID., "L'Art Mineur français et le curriculum grammatical", Histoire, Epistémologie, Langage 12:2, 1990, 15-29; ID. \& A. Dalzell, "Les manuscrits de l'Art mineur en ancien et moyen français. Description des manuscrits; L'Art Mineur de Vatican, Bibliotheca apostolica vaticana, Vat. lat. MS 1479 - Édition", Archives et documents de la Société d'Histoire et d'Épistémologie des Sciences du langage II: 4, 1990, 27-44; M. Colombo, "La traduction-remaniement de l'Ars minor de Donat du manuscrit B.N. n.a.f. 4690. Introduction; Édition", ibid. 1-26. 
struzione). Questo tipo di descrizione grammaticale suggerisce, quindi, un modello di "traslazione per equivalenza semantica" che è orientato dal volgare al latino: l'insegnamento presuppone un ambiente nel quale il volgare serve d'appoggio all'acquisizione della "lingua colta".

È importante mettere in rilievo la presenza implicita di quel modello. La sua operazionalità è patente in tre tipi di fenomeni, ben attestati nei frammenti grammaticali latino-friulani.

1. L'enumerazione di elementi grammaticali del latino uniti alle corrispondenze volgari, come appare nell'elenco seguente di nomina partitiva:

\begin{tabular}{|c|c|c|c|}
\hline uterque & -traque & -trumque & dicitur l'un e l'atri \\
\hline neuter & $-\operatorname{tra}$ & -trum & dicitur né l'un né l'atro \\
\hline & -tra & -trum & dicitur o l'un o l'atro \\
\hline
\end{tabular}

2. La presentazione dei mezzi sintattici per i quali il latino, usando vari verbi, può rendere una sola costruzione del volgare. Cosi, il concetto "aver bisogno di“" può ricevere cinque espressioni diverse in latino:

Nota quod cum datur tale thema Pieri abisugna di dinas latinum potest fieri quinque modis. Primo modo, per hoc verbum egeo, -es, et tunc vult habere post se genitivum vel ablativum sine preposicione, ut "ego egeo denarijs" vel "denariorum". Secondo modo, potest construi hoc verbum oporteo, -es, et tunc persona agens debet poni in nominativo et paciens in accusativo, ut "denarij oportent Petrum". Tercio modo, potest fieri latinum per hoc verbum sum, es et per hoc nomen opus vel necesse, et tunc latinum potest fieri dupliciter: primo modo, ponendo personam que habet indigenciam in dativo et rem que facit indigenciam in nominativo cum hoc nomine opus vel necesse, ut "denarij sunt opus vel necesse Petro"; secundo modo, potest fieri latinum per hoc verbum sum, es, est et per hoc nomen opus, vel necesse, ponendo personam que habet indigenciam in dativo [et rem] que facit indigenciam in genitivo vel in ablativo sine preposicione, ut "Petro est opus" vel "necesse denarijs" vel "denariorum"; et tunc opus declinatur: nominativo [habet] opus, accusativo hoc, et non habet plus. Quinto et ultimo modo, potest fieri latinum per hoc verbum sum, es, est et per hoc nomen necessarius, -ria, rium, ponendo rem que facit indigenciam in nominativo et personam que habet indi[genciam] in dativo, ut "denarij sunt necessarij Cançulo“.

(Schiaffini 1921: 99-100).

3. La distinzione di varie costruzioni del medesimo verbo latino con riferimento alle corrispondenze nel volgare, dove si fa uso di verbi distinti:

Nota quod hoc verbum ardeo, -es quatrupliceter potest construi. Uno modo, in quantum habet istud vulgare "ego ardeo" io m'inamor, et tunc vult habere an- 
te se nominativum et post se accusativum cum preposicione in, ut "ego ardeo in Bertam“. Secundo modo, potest construi hoc verbum ardeo, -es in quantum habet istud vulgare "ego ardeo" io am ardentmentri, et tunc vult determinari per accusativum sine preposicione, ut in Suma Magistri Cesaris ${ }^{5}$ "Coridon ardebat Alexim", id est, "ardenter amabat eam". Tercio modo, potest construi hoc verbum ardeo, -es in quantum stat per arder, et tunc vult habere ante se nominativum persone pacientis et post se ablativum persone agentis, ut "me ardent bona ligna“. Quarto et ultimo modo, potest construi hoc verbum ardeo, -es in quantum stat per desidrar, et tunc vult determinari per infinitivum, ut in Rigeto ${ }^{6}$ : "Me sibi privignum Ramusia dira naverca [sic] ardet in orendis et perpetuare malis"

(Schiaffini 1921: 102).

Occorre tenere a mente che il tipo di produzione grammaticale illustrato dai frammenti latino-friulani procede in modo analitico e per corrispondenza dialettica: nella descrizione delle costruzioni verbali, l'articolazione generale è ripresa dai modelli latini ("De verbis", "De participiis") e la divisione interna si conforma alle distinzioni tradizionali (verba activa, verba passiva, verba deponentia), ma la spiegazione - quindi, anche l'assimilazione da parte dell'allievo - di strutture si basa sulle rispondenze nel volgare. La corrispondenza talvolta consiste nella similarità di strutture sintagmatiche legata a un'opposizione di strutture morfologiche:

Nota quod verbum passivum est illud quod desinit in -or et descendit [ab hoc] verbo activo quod est amo, -as et doceor, -eris descendit ab hoc verbo activo quod est doceo, -es, et similia. Et est sciendum quod omne verbum passivum debet habere ante se nominativum et post se ablativum, a vel ab mediante, ex natura actus illati ab altero. Et persona agens debet poni in ablativo, a vel ab mediante, et paciens in nominativo. Scilicet:

$\begin{array}{llll}\text { amor } & \text {-aris } & \text { per } & \mathrm{v}(\text { ignir) amat } \\ \text { doceor } & \text {-eris } & \text { per } & \mathrm{v}(\text { ignir) amagistrat } \\ \text { legor } & \text {-eris } & \text { per } & \mathrm{v}(\text { ignir) let } \\ \text { audior } & \text {-iris } & \text { per } & \mathrm{v}(\text { ignir) audit } \\ \text { feror } & \text {-eris } & \text { per } & \mathrm{v}(\text { ignir) portat } \\ \text { diligor } & \text {-eris } & \text { per } & \mathrm{v}(\text { ignir) benvuglut } \\ \text { scribor } & \text {-eris } & \text { per } & \mathrm{v}(\text { ignir) scrit } \\ \text { concludor } & \text {-eris } & \text { per } & \mathrm{v}(\text { ignir) provat }\end{array}$

5 Il magister Cesar è l'autore del Compendium grammaticae pubblicato da Ch. Fierville, Une grammaire latine inédite du XIIF siècle, Paris, 1886; cf. p. 37: "verbum absolutum potest regere accusativum per figuram que dicitur sinodoche, ut "doleo caput"; ex vi verbi subintellecti, ut "Coridon ardebat Alexim", id est ardenter amabat".

6 Arrigo da Settimello, Arrighetto ovvero Trattato contro all'avversità della fortuna (ed. O. Manni, Firenze, 1730), p. 13: "Me sic privinum Rhamnusia dira noverca//Ardet in horrendis perpetuare malis". 
Et alia infinita que scribi non possunt. Ut in hoc exemplo Io [v]in amat del mestri "ego amor a magistro"

(Schiaffini 1921: 15).

Nei frammenti latino-friulani la costruzione dei verbi latini nella loro correlazione con verbi sinonimi del volgare, viene trattato in termini che riflettono l'elaborazione medievale della grammatica latina antica. Della grammatica romana si ritiene la classificazione generale in verba activa, passiva, deponentia, neutra, communia, basata sulle marche morfologiche ${ }^{7}$ e sul criterio sintattico di combinazione con un oggetto diretto (all'accusativo). Dalla grammatica medievale derivano i concetti, sintattici, di costruzione transitiva e costruzione intransitiva, e quelli, semantici, di persona agens e patiens. L'influenza della grammatica medievale (tanto della grammatica didattica quanto della grammatica speculativa ) ̀̀ anche visibile nel riferimento alla posizione degli elementi governati dal verbo: il concetto di regere a parte ante si riferisce al componente soggetto e tutti i casi obliqui sono riportati al concetto di regere a parte post ${ }^{8}$.

Non occorre cercare grande originalità in questi tipi di testi didattici; tuttavia, $\mathrm{i}$ frammenti latino-friulani presentano, mi pare, due aspetti interessanti, su cui vorrei soffermarmi. Il primo è quello di una riflessione sullo status dei concetti persona agens o patiens: questi non sono legati in modo meccanico al nominativo della frase attiva (o all'ablativo dell'agente) e all'accusativo (o al nominativo della frase passiva). Nei frammenti osserviamo che si dà la precedenza a una concezione semantica pura, quando viene discusso il caso dei verbi metuere e timere:

Et est sciendum quod omne verbum activum debet habere ante se nominativum et post se accusativum. Et persona agens debet poni in nominativo et [persona paciens] in accusativo, ut in hoc exemplo io am $\mathrm{Ca}[\mathrm{n}]$ çul "ego amo Cançolum", preterquam in istis duobus verbis que sunt metuo $\langle\mathrm{r}$ ' $\rangle$ timeo, in quibus persona agens debet poni in accusativo et paciens in nominativo, ut in hoc exemplo io tim Jaculin "ego timeo" vel "metuo Cançulum".

(Schiaffini 1921: 10).

Il secondo aspetto interessante di questi frammenti risiede nella concezione della frase come struttura sintattica con valore di predicazione determinata. In una bre-

7 "Nota quod omne verbum quod desinit in -o aut est verbum activum aut neutrum. Verbum neutrum est illud quod desinit in -o et non habet passivum, ut ovio, gaudeo, vado et similia. Verbum activum est illud quod desinit in -o et habet passivum ut amo, -as habet passivum, scilicet amor, -ris, et doceo, -es habet passivum, scilicet doceor, -ris" (Schiaffini 1921: 10).

8 La costruzione passiva si caratterizza per la conversione dei ruoli semantici rispetto a queste posizioni: "Nota quod omne verbum passivum potest regere a parte ante et a parte post il[lum] casum quem regit suum activum preter [l. propter] ejis propriam determinacionem, ut "ego do tibi panem" et "panis datur tibi a me". Et est sciendum quod ista sunt quedam verba passiva que debent habere ante se nominativum et post se ablativum, a vel ab mediante, [per] propriam naturam et dativum per naturam communem repertam in verbo passivo et in suo activi. Et persona agens debet poni in ablativo, a vel ab mediante, et paciens in nominativo. Scilicet: daris vel dare/ datur per v(ignir) dat; comparor -aris per v(ignir) asemeglat“" (Schiaffini 1921: 15-16). 
ve sezione l'autore fa la distinzione tra oratio infinita (cioè un contenuto proposizionale) e oratio determinata (una frase concreta, con valore di verità determinato). Per avere una frase "determinata", occorre un operatore sentenziale (che conferisce valore esistenziale al contenuto proposizionale) e un "operatore" con valore referenziale: in questo modo si passa dal contenuto "qualcuno-venire" all'enunciato " $\mathrm{X}$ viene/è venuto/verrà“.

Tercio modo potest fieri latinum sic et melius et est "quod ego veni", ita quod illa oratio "quod ego veni", infinitata istam coniunctionem infinitam "quod", veniat ad suposicionem del "est" et lu "tres dies" ad determinacionem orationis. Item cum datur tale tema Pieri vens trey dis pasaç, latinum potest fieri per concomitanciam sic: "Petrus venit tribus diebus elapsis".

(Schiaffini 1921: 99).

In questo passo incontriamo in nuce la distinzione moderna tra enunciazione e proposizione: è interessante osservare che in un testo di carattere didattico e di stampo tradizionale appaiono non solo strategie d'innovazione descrittiva (per la corrispondenza stabilita con il volgare), ma anche concezioni teoriche che trascendono il quadro pedagogico e legano la grammatica alla filosofia. Ciò rappresenta la conferma dello status interdisciplinare della grammatica nel Medio Evo e, allo stesso tempo, la prova del valore e della rilevanza di testi immersi in una cultura di confronto tra latino e l'eredità classica di un lato, e volgare e il pensiero scolastico dall'altro.

\author{
Povzetek
}

O NEKATERIH NAČELIH SESTAVLJANJA SLOVNIC V LJUDSKEM ROMANSKEM JEZIKU: LATINSKO-FURLANSKI ODLOMKI

Latinsko-furlanski odlomki, didaktični teksti iz 14. st., predstavljajo neko metodo učenja slovnice, ki temelji na "primerjavi pomenskih enakovrednosti“. Ta postopek je viden v teh pojavih: 1) naštevanje latinskih slovničnih enot povezanih z ustreznimi enotami v ljudskem jeziku; 2) predstavitev skladenjskih sredstev, s katerimi latinščina lahko izrazi konstrukcijo iz ljudskega jezika; 3) navajanje različnega konstruiranja $v$ latinščini in $v$ romanskem ljudskem jeziku pri enem in istem glagolu.

Latinsko-furlanski odlomki odsevajo preživetje antičnih slovničnih vzorcev, dopolnjeni pa so z dognanji srednjeveške gramatike. Če ta didaktični priročnik, splošno vzeto, ni preveč inovativen, pa vendar kaže vsaj dva zanimiva vidika: a) razmišljanje, in sicer semantične narave, o statutu pojma delujoča ose$b a$ in oseba, ki akcijo trpi in b) razlikovanje med oratio infinita (abstraktno vsebino stavka) in oratio determinata (konkretnim stavkom $z$ vrednostjo enunciacije). 